\title{
Successful Treatment with Rituximab for Granulomatosis with Polyangiitis and Multiple Cranial Neuropathies
}

\author{
Kazuhiro Horiuchi, Yuki Oshima and Akihiko Kudo
}

\begin{abstract}
:
We herein report a case of granulomatosis with polyangiitis in a 73-year-old man. He had experienced bilateral ptosis, redness of both eyes, right facial weakness, and hearing loss in the right ear for two months. Myeloperoxidase anti-neutrophil cytoplasmic antibody was positive. Corticosteroids and intravenous cyclophosphamide pulse therapy yielded a response. After the fourth pulse of cyclophosphamide, he developed headache, diplopia, restricted left eye movement, right facial palsy, and hearing loss in the right ear. A oneyear remission period was achieved by increasing the steroid and rituximab doses. Rituximab was effective against the relapse of granulomatosis with polyangiitis and cranial neuropathy.
\end{abstract}

Key words: granulomatosis with polyangiitis, MPO-ANCA, rituximab, cyclophosphamide, cranial neuropathy

(Intern Med 60: 1095-1099, 2021)

(DOI: 10.2169/internalmedicine.4881-20)

\section{Introduction}

Granulomatosis with polyangiitis (GPA) is an autoimmune vasculitis of small-to-medium-sized blood vessels. The classic or complete form of GPA affects the upper respiratory tract, lungs, and kidneys. It can also occur in the limited form, which usually involves the head and neck. The Chapel Hill 2012 Consensus Criteria define GPA as "necrotizing granulomatous inflammation usually involving the upper and lower respiratory tract and necrotizing vasculitis affecting predominantly small to medium vessels"(1).

Treatments for GPA involve intensive immunosuppressive therapy with high-dose corticosteroids and cyclophosphamide or rituximab to induce remission. Once remission is achieved, patients are switched to less-toxic maintenance immunosuppression, such as azathioprine and low-dose corticosteroid (2). Localized GPA typically manifests with middle ear and respiratory tract features such as otitis media, sinusitis, nasal collapse, and airway stenosis.

We herein report a case of localized GPA that relapsed, presented with progressive and multiple cranial nerve palsies, and responded to rituximab treatment.

\section{Case Report}

The patient was a 73-year-old man with GPA who had no previous medical history. His family history was unremarkable. He had experienced bilateral ptosis, redness of both eyes, right facial palsy, and hearing loss in the right ear for two months. He was referred to our institution because the symptoms continued to progress.

On an examination, he appeared ill, with a pulse rate of 72 beats/min; blood pressure, $124 / 74 \mathrm{mmHg}$; respiratory rate, 10 breaths/min; oxygen saturation, 96\%; and temperature, $37.0^{\circ} \mathrm{C}$. His height was $157 \mathrm{~cm}$, weight $50 \mathrm{~kg}$. His eyes were congested, and he had pain in both eyes and face. Saddle nose deformity was not observed. Breathing sounds were clear on chest auscultation. Body edema was not observed. On a neurological examination, he was alert and showed bilateral hyposmia, a decreased light reflex in the right eye, bilateral ptosis, right eye abduction limitation, diplopia (right lateral gaze), reduced superficial sensation at the upper right part of the face, right facial muscle weakness, dysgeusia, and mild bilateral hearing loss.

Initial laboratory studies showed the following: a white blood cell count of $6,200 / \mathrm{mm}^{3}$ (neutrophils $79.8 \%$, lymphocytes $14.8 \%$, monocytes $4.7 \%$, eosinophils $0.5 \%$, and baso- 


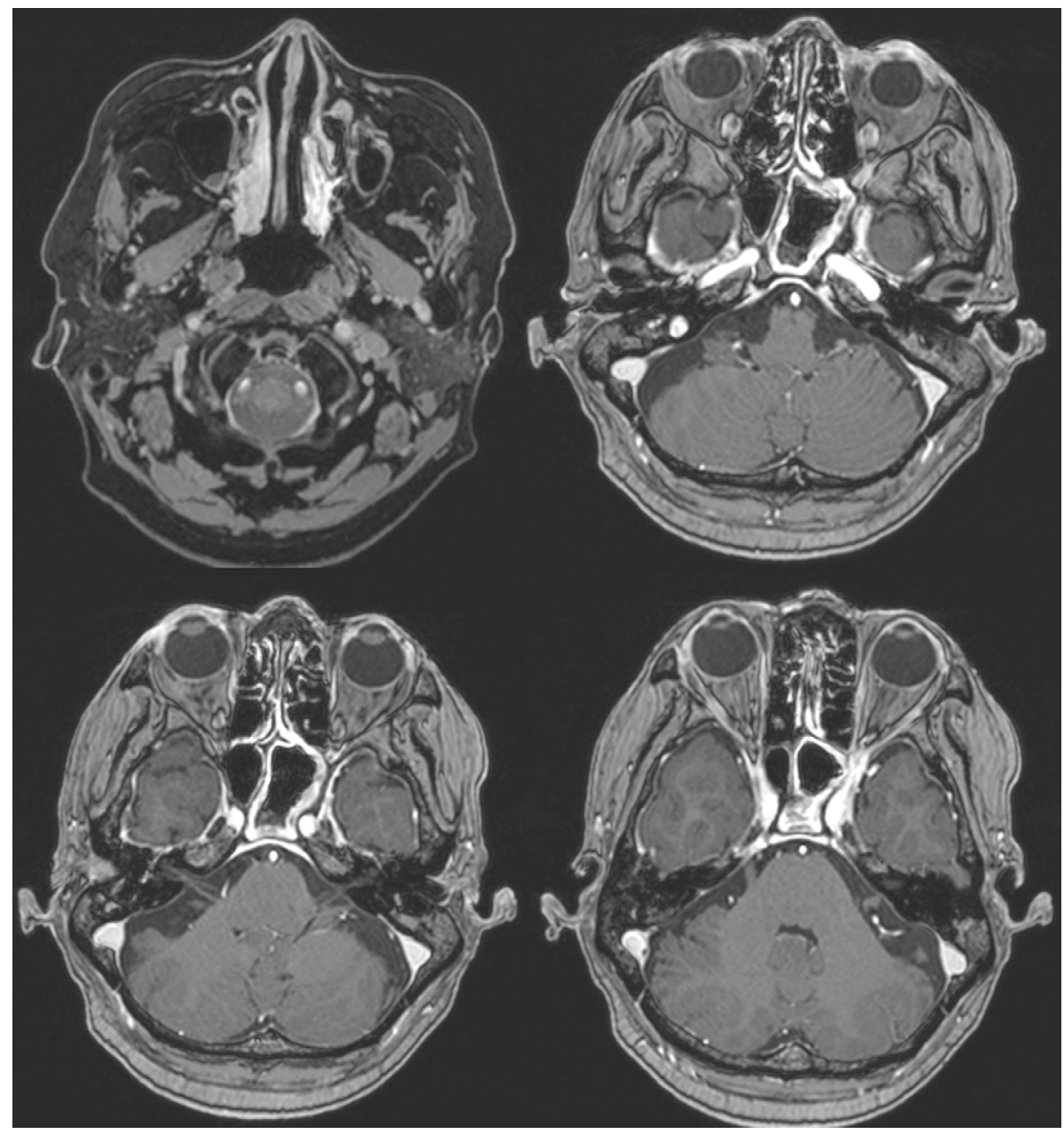

Figure. Brain MRI with T1 gadolinium enhancement (TR 9 TE 3.4) showed dural thickening in both temporal lesions, indicating pachymeningitis. In the paranasal sinuses, mucosal thickening of the maxillary sinuses, minimal fluid retention, and bone wall thickening were observed, suggesting chronic sinusitis. Inflammation of the bilateral ocular conjunctiva was also observed. Overall, the findings were indicative of right-dominant bilateral otitis media.

phils $0.2 \%$ ), hemoglobin $13.0 \mathrm{~g} / \mathrm{dL}$, platelet count $446 \times 10^{3} /$ $\mathrm{mm}^{3}$, C-reactive protein (CRP) $6.22 \mathrm{mg} / \mathrm{dL}$, aspartate aminotransferase 13 international units (IU)/L, alanine aminotransferase $12 \mathrm{IU} / \mathrm{L}$, blood urea nitrogen $12.5 \mathrm{mg} / \mathrm{dL}$, creatinine $0.55 \mathrm{mg} / \mathrm{dL}$, sodium $140 \mathrm{mEq} / \mathrm{L}$, potassium 4.3 $\mathrm{mEq} / \mathrm{L}$, and chloride $102 \mathrm{mEq} / \mathrm{L}$, glucose, $99 \mathrm{mg} / \mathrm{dL}$, HbA1c, 6.1\%; IgG, $926 \mathrm{mg} / \mathrm{dL}$, prothrombin time $11.2 \mathrm{sec}-$ onds, international normalized ratio (INR), 0.9 (normal range $<1.15$ ), activated partial thromboplastin time $28.7 \mathrm{sec}-$ onds (normal range $<42 \mathrm{~s}$ ), rheumatoid factor $10 \mathrm{IU} / \mathrm{mL}$ (normal range $<15 \mathrm{IU} / \mathrm{mL}$ ), proteinase 3 anti-neutrophil cytoplasmic antibody (PR3-ANCA) $<1.0 \mathrm{U} / \mathrm{mL}$ (normal value $<3.5 \mathrm{U} / \mathrm{mL}$ ), myeloperoxidase anti-neutrophil cytoplasmic antibody (MPO-ANCA) $4.8 \mathrm{U} / \mathrm{mL}$ (normal range $<3.5 \mathrm{U} /$ $\mathrm{mL}$ ), angiotensin-converting enzyme $8.1 \mathrm{U} / \mathrm{L}$ (normal range 8.3-21.4 U/L), soluble interleukin-2 receptor $496 \mathrm{U} / \mathrm{mL}$, and $\beta$-D-glucan $<6 \mathrm{pg} / \mathrm{mL}$. Cryptococcus neoformans antigens and aspergillus antigens were both negative. Anti-Sjögren's syndrome (SS)-A, SS-B, ribonucleoprotein, double-stranded DNA immunoglobulin $\mathrm{G}$, and cyclic citrullinated peptide antibodies were not detected. A urinalysis showed no urine protein or occult blood. In the cerebrospinal fluid (CSF) test, the lumbar CSF opening pressure was $130 \mathrm{mmH}_{2} \mathrm{O}$,

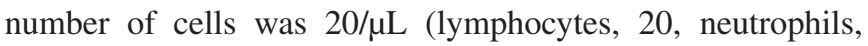
0), protein level was $80.8 \mathrm{mg} / \mathrm{dL}$, glucose level was $59 \mathrm{mg} /$ $\mathrm{dL}$, IgG level was $19.0 \mathrm{mg} / \mathrm{dL}$, IgG index was 2.08, and myelin basic protein level was $<40 \mathrm{mg} / \mathrm{dL}$. An oligoclonal band was not detected.

Chest radiographs and computed tomography (CT) images were normal. Brain magnetic resonance imaging (MRI) revealed dural thickening in both temporal lesions, but the trigeminal, facial, and auditory nerves showed no gadolinium enhancement. In the paranasal sinuses, mucosal thickening of the maxillary sinuses, minimal fluid retention, and bone wall thickening were detected, indicating chronic sinusitis. Inflammation of the bilateral ocular conjunctiva was also observed. Overall, the findings were indicative of rightdominant bilateral otitis media (Figure).

Both sides of the mastoid were poorly aerated, with soft opacity in the tympanic cavity and evidence of otitis media. A consultation with an ophthalmologist for the redness of both eyes resulted in a diagnosis of scleritis. We performed 
a biopsy of the thickened nasal mucosa. At the biopsy site of the sinus mucosa, numerous eosinophils, neutrophils, and plasma cells were found in addition to capillaries with thickened basement membranes and vasculogenesis. We observed no evidence of fibrinoid necrosis, other vasculitis, or perivascular inflammation that would clearly indicate vasculitis. In addition, no fungal infection or malignancy was found. Based on the otitis media, scleritis, hypertrophic pachymeningitis, and PR3-ANCA-positive findings, the patient was diagnosed with localized GPA (MPO-ANCA-positive). Furthermore, according to the algorithm of the European Medicines Agency, this case is confirmed by the GPA for the diagnosis of ANCA-related vasculitis (3).

The patient was started on treatment with 2 courses of intravenous pulses of methylprednisolone $1,000 \mathrm{mg} /$ day for 3 days, which resulted in rapid improvement of bilateral ptosis, right eye abduction limitation, diplopia, reduced superficial sensation in the upper right side of the face, dysgeusia, and bilateral hearing loss. CRP and MPO-ANCA became negative. However, the bilateral hyposmia, decreased righteye light reflex, and facial muscle weakness on his right side remained. His oral prednisolone dose was $50 \mathrm{mg} /$ day after intravenous methylprednisolone (IVMP) 1,000 mg/day for 3 days, and he received 4 doses of intravenous cyclophosphamide $500 \mathrm{mg}(10 \mathrm{mg} / \mathrm{kg})$ at monthly intervals. The dose of prednisolone was gradually tapered to $15 \mathrm{mg} /$ day.

Four weeks after the fourth pulse of cyclophosphamide, he developed a headache, diplopia, restricted left-eye movement in all directions, weakness of the right facial muscles, and exacerbation of hearing loss in the right ear. No symptoms other than cranial nerve issues and headache were observed. The levels of MPO-ANCA (2.8 U/mL) and CRP (3.52 $\mathrm{mg} / \mathrm{dL})$ were increased, and PR3-ANCA was absent. Gadolinium-enhanced brain MRI detected no evidence of cerebral nerve thickening. However, it did reveal persistent mucosal thickening of the sinus and pachymeningitis despite improvement in the symptoms of sinusitis and otitis media. We therefore diagnosed the patient with localized GPA relapse and reinitiated methylprednisolone at $1,000 \mathrm{mg} /$ day for 3 days.

Prednisolone was increased to $50 \mathrm{mg} /$ day, and rituximab was started because intravenous cyclophosphamide monthly was not sufficiently effective. Rituximab was administered at $375 \mathrm{mg} / \mathrm{m}^{2}$ weekly for a total of 4 doses. His headache soon improved. Diplopia, eye movement restriction, right facial muscle weakness, and right hearing loss gradually improved. No side effects of rituximab were observed. The patient was switched to maintenance therapy with azathioprine for 6 months after completing the rituximab course, and the prednisolone dose was slowly tapered to $5 \mathrm{mg}$ /day. He remained in good clinical condition except for mild residual weakness of the right facial muscles for one year following the completion of rituximab.

\section{Discussion}

Central nervous system complications are common in patients with GPA. In a European cohort of 128 GPA patients, $56(44 \%)$ had peripheral neuropathy (either symmetrical polyneuropathy or mononeuritis multiplex). Only 9 patients (7\%) had CNS involvement, including 6 with cranial nerve palsies (4). CNS involvement occurred in $8 \%$ of cases in a cohort from the National Institutes of Health (5), and cranial nerve palsies occurred in 21 of 324 patients (6\%) (6). CNS disease in GPA is attributed to three distinct but sometimes co-existing mechanisms: by spread of granulomatous tissue from adjacent sites in the middle ear and sinuses (as in our patient), by primary granuloma formation within the CNS, or by vasculitis affecting the CNS. In this case, there were no findings on MRI suggestive of vasculitis. The arteries affected by GPA were considered to be consistent with the fact that arteries of the size generally seen on MRI were not affected.

Several previous cases of hearing symptoms, along with granulomatous nose, throat, and eye were diagnosed with GPA and treated with rituximab (7). Facial palsy associated with otitis media is a well-known common feature of PR3ANCA-related granulomatosis; however, cranial polyneuropathies are also possible in MPO-ANCA-related vasculitis, albeit with a lower reported incidence (8-10).

Although the number of PR3-ANCA-positive patients was small, a previous study found a higher frequency of pulmonary and renal disease in PR3-ANCA-positive cases than in MPO-ANCA-positive cases, with leptomeningeal and parenchymal involvement in the brain being more common in PR 3-positive hypertrophic pachymeningitis than in MPOpositive and idiopathic hypertrophic pachymeningitis (11). In our study, no contrast findings or thickening of the cranial nerves was detected by MRI; however, we concluded the patient's diagnosis to be multiple cranial neuropathies caused by hypertrophic pachymeningitis. No lung or kidney lesions occurred, and relapse only manifested as cranial neuropathies. Thickening of the maxillary sinus mucosa was observed by CT and MRI, but thickening of the cranial nerves could not be detected by MRI. There have been reports of dysphagia and pituitary disorders in patients with localized GPA (12). It is possible that if the patient's symptoms had progressed, dysphagia may have occurred, and lung and kidney disorders may have appeared. In localized GPA, 15 out of $34(44 \%)$ patients were PR3-ANCA-positive (>6 IU/mL) before treatment and 23/34 (68\%) were positive for CANCA. Flare-ups during follow-up were unreliable (range 20-100\%), and the specificity was more consistent (range 83-90\%). All three patients with elevated MPO-ANCA levels became negative after treatment with rituximab, and all remained negative during follow-up. One of the three patients subsequently had disease recurrence without an increase in MPO-ANCA (7). In a Japanese cohort study, MPO-ANCA was positive in $62.3 \%$ of patients with 
GPA (13). Furthermore, seronegative GPA presenting with multiple cranial nerve palsies was reported (14). Although MPO-ANCA was positive in our case, we diagnosed it as GPA based on the clinical symptoms and pathological findings.

According to a previous study on refractory GPA, rituximab therapy resulted in complete remission/improvement in $89.2 \%$ of patients with renal disease and $80.8 \%$ with alveolar hemorrhaging. However, for orbital mass and pachymeningitis cases, rituximab therapy resulted in improvement in only $44.4 \%$ and $49.9 \%$ of patients, respectively (15), suggesting that rituximab may be more effective for treating vasculitis than for granulomatous manifestations. Furthermore, orbital pseudotumor and pachymeningitis may cause scarring instead of ongoing inflammation; meanwhile, rituximab was effective in cranial neuropathies caused by granuloma lesions (16). Regarding the effects of rituximab on ANCA-associated vasculitis, most studies have reported > $80 \%$ complete or partial remission rates, with the lowest response $(37.5 \%)$ obtained for granulomatous lesions (17). In the current case, hypertrophic pachymeningitis at the site of multiple cranial neuropathies did not lead to progressive scarring due to long-term inflammation. We believe that rituximab might have been effective if it had been administered before irreversible scarring changes occurred.

The RAVE and RITUXIVAS studies both showed the non-inferiority of rituximab compared to cyclophosphamide in AAV $(18,19)$. However, the results from these trials cannot necessarily be applied to our patient. The RITUXIVAS trial and RAVE studies did not have any patients with cranial nerve palsies in the rituximab treatment arm. Nevertheless, case series suggest that rituximab can be effective against cranial neuropathy of $\operatorname{GPA}(7,20)$. From 1985 to $2003,23 \%$ of 350 patients with ANCA-associated vasculitis exhibited treatment resistance. A low glomerular filtration rate, female sex, MPO-ANCA positivity, older age, and chronic kidney biopsy histology have been shown to be risk factors for treatment resistance (21). The present case met the following two criteria: positive for MPO-ANCA and older age. Although IVMP was effective, we reduced the amount of prednisolone taken to $15 \mathrm{mg} /$ day at 4 months after treatment initiation to avoid infections. Combining highdose steroids and cyclophosphamide had a high probability of resulting in concomitant infections; thus, the prednisolone dose was reduced. Relapse occurred because the standard dose of intravenous cyclophosphamide (IVCY) was reduced from $15 \mathrm{mg} / \mathrm{kg}$ to $10 \mathrm{mg} / \mathrm{kg}$ every 4 weeks. When recurrence occurred, we could have responded by increasing the dose of prednisolone and IVCY, but the patient was relatively old (73 years old), so we stopped IVCY administration and initiated rituximab therapy to prevent side effects such as infections.

Regarding rituximab therapy, 3 of 28 responders (11\%) followed for at least 12 months had relapsed by that time point. A further 16 had to repeat rituximab treatment due to an incomplete response or early symptoms suggestive of re- lapse but without sufficient disease activity. All patients responded after repeated rituximab treatment, which was evidenced by no further relapses and a decreased dose of glucocorticoids. At 18 months of follow-up, a further 2 patients had suffered disease relapse $(2 / 17,12 \%)(7)$. As demonstrated by several clinical trials, induction remission therapy with rituximab, in which prednisolone was administered for 14 weeks, resulted in favorable outcomes, with $48 \%$ of the patients maintaining remission at 12 months and $36 \%$ maintaining it at 18 months (22). In addition, maintenance remission therapy with rituximab once every 6 months has been shown to lead to a positive response (23).

Rituximab is commonly administered at $375 \mathrm{mg} / \mathrm{m}^{2}$ once a week for 4 weeks; however, in few cases, rituximab administration at $375 \mathrm{mg} / \mathrm{m}^{2}$ once only and initial prednisolone at $20 \mathrm{mg} /$ day successfully induced remission and maintained patient safety (24). In the present case, rituximab rapidly improved the symptoms during relapse. In elderly patients, adjusting the number of rituximab doses and concomitant prednisolone doses over the course of the treatment may reduce the risk of infection. The remission rates for rituximab and cyclophosphamide for vasculitis are similar, but some patients are contraindicated for cyclophosphamide therapy or refuse such therapy because of concerns about myelosuppression, fertility, hair loss, and malignancy risk. Furthermore, the use of high-dose steroids is challenging for people with severe diabetes and osteoporosis. High-dose steroids and cyclophosphamide induction can be avoided by considering the first-line induction of rituximab and low-dose steroids during remission or maintenance.

\section{Conclusion}

We herein report a case of local GPA presenting mainly with cranial neuropathy symptoms. Rituximab treatment was effective in relieving the cranial neuropathy induced by GPA when relapse occurred with cyclophosphamide or corticosteroid treatment.

The authors state that they have no Conflict of Interest (COI).

\section{Acknowledgement}

We would like to thank the staff of the medical unit for their help in the management of this patient.

\section{References}

1. Jennette JC, Falk RJ, Bacon PA, et al. 2012 revised International Chapel Hill Consensus Conference Nomenclature of Vasculitides. Arthritis Rheum 65: 1-11, 2013.

2. Yates M, Watts RA, Bajema IM, et al. EULAR/ERA-EDTA recommendations for the management of ANCA-associated vasculitis. Ann Rheum Dis 75: 1583-1594, 2016.

3. Watts R, Lane S, Hanslik T, et al. Development and validation of a consensus methodology for the classification of the ANCAassociated vasculitis and polyarteritis nodosa for epidemiological studies. Ann Rheum Dis 66: 222-227, 2007.

4. de Groot K, Schmidt DK, Arlt AC, Gross WL, Reinhold-Keller E. Standardized neurologic evaluations of 128 patients with Wegener 
granulomatosis. Arch Neurol 58: 1215-1221, 2001.

5. Hoffman GS, Kerr GS, Leavitt RY, et al. Wegener granulomatosis: an analysis of 158 patients. Ann Intern Med 116: 488-498, 1992

6. Nishino H, Rubino FA, DeRemee RA, Swanson JW, Parisi JE. Neurological involvement in Wegener's granulomatosis: an analysis of 324 consecutive patients at the Mayo Clinic. Ann Neurol 33: 4-9, 1993.

7. Martinez Del Pero M, Chaudhry A, Jones RB, Sivasothy P, Jani P, Jayne D. B-cell depletion with rituximab for refractory head and neck Wegener's granulomatosis: a cohort study. Clin Otolaryngol 34: 328-335, 2009.

8. Yoshida N, Iino Y. Pathogenesis and diagnosis of otitis media with ANCA-associated vasculitis. Allergol Int 63: 523-532, 2014.

9. Yoshida N, Hara M, Hasegawa M, et al. Reversible cochlear function with ANCA-associated vasculitis initially diagnosed by otologic symptoms. Otol Neurotol 35: 114-120, 2014.

10. Ferri E, Armato E, Capuzzo P, Cavaleri S, Ianniello F. Early diagnosis of Wegener's granulomatosis presenting with bilateral facial paralysis and bilateral serous otitis media. Auris Nasus Larynx 34: 379-382, 2007.

11. Yokoseki A, Saji E, Arakawa M, et al. Hypertrophic pachymeningitis: significance of myeloperoxidase anti-neutrophil cytoplasmic antibody. Brain 137: 520-536, 2014.

12. Peters JE, Gupta V, Saeed IT, Offiah C, Jawad ASM. Severe localised granulomatosis with polyangiitis (Wegener's granulomatosis) manifesting with extensive cranial nerve palsies and cranial diabetes insipidus: a case report and literature review. BMC Neurol 18: 59, 2018.

13. Sada KE, Harigai M, Amano $\mathrm{K}$, et al. Comparison of severity classification in Japanese patients with antineutrophil cytoplasmic antibody-associated vasculitis in a nationwide, prospective, inception cohort study. Mod Rheumatol 26: 730-737, 2016.

14. Lee E, Park J, Choi SH, Park SH. Seronegative granulomatosis with polyangiitis presenting with multiple cranial nerve palsies. Neuropathology 38: 192-197, 2018.

15. Holle JU, Dubrau C, Herlyn K, et al. Rituximab for refractory granulomatosis with polyangiitis (Wegener's granulomatosis): comparison of efficacy in granulomatous versus vasculitic manifestations. Ann Rheum Dis 71: 327-333, 2012.

16. Nakazawa M, Suzuki K, Yasuoka H, Yamaoka K, Takeuchi T. Successful rituximab treatment of granulomatosis with polyangiitis with cranial neuropathies. Inflamm Regen 38: 20, 2018.

17. Ayan G, Esatoglu SN, Hatemi G, et al. Rituximab for antineutrophil cytoplasmic antibodies-associated vasculitis: experience of a single center and systematic review of non-randomized studies. Rheumatol Int 38: 607-622, 2018.

18. Stone JH, Merkel PA, Spiera R, et al. Rituximab versus cyclophosphamide for ANCA-associated vasculitis. N Engl J Med 363: 221-232, 2010.

19. Jones RB, Tervaert JW, Hauser T, et al. Rituximab versus cyclophosphamide in ANCA-associated renal vasculitis. N Engl J Med 363: 211-220, 2010.

20. Guerry MJ, Brogan P, Bruce IN, et al. Recommendations for the use of rituximab in anti-neutrophil cytoplasm antibody-associated vasculitis. Rheumatology (Oxford) 51: 634-643, 2012.

21. Hogan SL, Falk RJ, Chin H, et al. Predictors of relapse and treatment resistance in antineutrophil cytoplasmic antibody-associated small-vessel vasculitis. Ann Intern Med 143: 621-631, 2005.

22. Specks U, Ikle D, Stone JH. Induction regimens for ANCAassociated vasculitis. N Engl J Med 369: 1865-1866, 2013.

23. Smith RM, Jones RB, Guerry MJ, et al. Rituximab for remission maintenance in relapsing antineutrophil cytoplasmic antibodyassociated vasculitis. Arthritis Rheum 64: 3760-3769, 2012.

24. Turner-Stokes T, Sandhu E, Pepper RJ, et al. Induction treatment of ANCA-associated vasculitis with a single dose of rituximab. Rheumatology (Oxford) 53: 1395-1403, 2014.

The Internal Medicine is an Open Access journal distributed under the Creative Commons Attribution-NonCommercial-NoDerivatives 4.0 International License. To view the details of this license, please visit (https://creativecommons.org/licenses/ by-nc-nd/4.0/).

(C) 2021 The Japanese Society of Internal Medicine Intern Med 60: 1095-1099, 2021 